\title{
Seroprevalencia del virus linfotrópico humano de tipos I y II en donantes del Banco de Sangre de la Fundación Valle del Lili, Cali, Colombia, 2008-2014
}

\author{
Carmenza Macía ${ }^{1}$, Sandra Vargas ${ }^{1}$, Ana María Mora², Ashly Melissa Sarmiento², \\ Robinson Pacheco ${ }^{3}$, Fernando Rosso ${ }^{3}$ \\ Banco de Sangre Fundación y Servicio Transfusional (sic), Clínica Valle del Lili, Cali, Colombia \\ 2 Facultad de Medicina, Universidad ICESI, Cali, Colombia \\ 3 Centro de Investigaciones Clínicas, Fundación Valle del Lili, Cali, Colombia
}

Introducción. El virus linfotrópico humano (HTLV) de tipos I y II es un retrovirus prevalente en la Costa Pacífica colombiana que puede transmitirse por transfusiones de sangre. En el 2014 se reglamentó la tamización para bancos de sangre con el fin de reducir la transmisión por medio de la donación. La información sobre la seroprevalencia del virus en el suroccidente colombiano es limitada.

Objetivo. Determinar la seroprevalencia, el comportamiento a lo largo del tiempo de los resultados reactivos antes y después de la introducción del inmunoensayo Western blot y la concomitancia del HTLV con otros marcadores de infección en donantes de un banco de sangre de Cali, Colombia.

Materiales y métodos. Se hizo un estudio trasversal de 77.117 donantes del Banco de Sangre de la Fundación Valle del Lili mediante el análisis de los registros de donantes con prueba reactiva para anticuerpos IgG anti HTLV I-II entre enero de 2008 y diciembre de 2014.

Resultados. La seroprevalencia acumulada fue de 0,24\%. Los resultados reactivos fueron más frecuentes en mujeres $(61 \%$ y la mediana de edad fue de 37 años. La seroprevalencia en los años previos a la introducción del Western blotfue de $0,13,0,19,0,31$ y $0,32 \%$ (2008-2012), y posteriormente fue de $0,18,0,08$ y $0,07 \%(2012-2014)$. La reacción positiva concomitante con otros marcadores de infección fue de $11 \%$ : sífilis (57\%), HIV (19\%), hepatitis B (14\%) y hepatitis C (9\%). La mayor seroprevalencia $(0,38 \%)$ se registró en el 2012.

Conclusión. Se encontró una alta prevalencia de pruebas reactivas para el HTLV I-II en comparación con otros estudios. Los resultados de este estudio son un punto de partida para el desarrollo de estudios poblacionales.

Palabras clave: anticuerpos anti-HTLV-I, anticuerpos anti-HTLV-II, donantes de sangre, prevalencia, serología, Western blotting.

doi: http://dx.doi.org/10.7705/biomedica.v36i0.2942

Seroprevalence of human T-lymphotropic virus in blood bank donors at Fundación Valle del Lili, Cali, Colombia, 2008-2014

Introduction: Human lymphotropic virus (HTLV I/II) is a retrovirus that is prevalent across the Colombian Pacific coast, and is potentially transmissible by transfusion. Blood bank screening has been regulated since 2004, in order to reduce transmission of HTLV I/II through donation. Information on the seroprevalence of the virus in southwestern Colombia is limited.

Objective: To determine the seroprevalence and the behavior of reactivity to HTLV I/II before and after the introduction of Western blot, and the comorbidity of HTLV and other infectious markers in donors from a blood bank in Cali, Colombia.

Materials and methods: We conducted a cross-sectional study of 77,117 blood bank donors from the Fundación Valle del Lili by analyzing records of donors who had been tested with the reactive test for anti-HTLV I-II antibodies (IgG) between January, 2008, and December, 2014.

Results: The cumulative seroprevalence during the study period was $0.24 \%(186 / 77,119)$. Reactivity was more common in women (61\%), and the median age was 37 years (IQR: 24-48). The seroprevalence in the years before the introduction of Western blot was $0.13 \%, 0.19 \%, 0.31 \%, 0.32 \%$ and $0.18 \%(2008-$

\section{Contribución de los autores:}

Robinson Pacheco y Fernando Rosso: desarrollo de la idea, diseño del estudio y revisión crítica del contenido

Carmenza Macía y Sandra Vargas: implementación de las pruebas de tamización y confirmatorias, creación de la base de datos, análisis de la información y revisión crítica del contenido

Ana María Mora y Ashly Melissa Sarmiento: desarrollo de la idea, revisión de la literatura, diseño del estudio y análisis de la información

Todos los autores participaron en la redacción del manuscrito. 
2012), and thereafter it was $0.08 \%$ and $0.07 \%$ (2012-2014). Concomitant reactivity with other infectious markers was $11 \%$ : syphilis $(57 \%)$, followed by HIV $(19 \%)$, hepatitis B $(14 \%)$ and hepatitis C $(9 \%)$. The highest seroprevalence $(0.38 \%)$ was reported in 2012.

Conclusion: We found a high prevalence of reactivity to HTLV I-II compared to that reported in other studies. The results of this study are a starting point for the development of population studies.

Key words: HTLV-I antibodies; HTLV-II antibodies; blood donors; prevalence; serology; blotting, Western. doi: http://dx.doi.org/10.7705/biomedica.v36i0.2942

El virus linfotrópico de células T humanas (Human T-cell Lymphotropic Virus, HTLV) pertenece a la familia Retroviridae, subfamilia Oncovirinae y al grupo de los virus linfotrópicos de primates (PTLV) (1). Se conocen cuatro subtipos (HTLV I-IV) y solo los subtipos HTLV I y II se han relacionado con el desarrollo de enfermedades en humanos (2-4). El HTLV-1 es un retrovirus intracelular cuyo blanco principal son los linfocitos T. La concentración de formas libres del virus en plasma es sumamente baja, por lo cual la transmisión se da a partir del contacto con linfocitos infectados (5). El virus se puede adquirir por diferentes vías: vertical (madre-hijo), contacto sexual y vía parenteral por transfusión sanguínea o uso de inyecciones e instrumentos de aplicación de medicamentos parenterales (6). El riesgo de adquirir la infección varía dependiendo de la vía de contagio. La vía más efectiva de trasmisión es la endovenosa, especialmente la transfusión de glóbulos rojos, seguida de la trasmisión por la lactancia, con una eficiencia de $20 \%$, y, por último, la vía intrauterina y durante el parto, con $5 \%$ (7).

Son diversas las enfermedades relacionadas con la infección por HTLV I-II, pero solo entre el 5 y el $10 \%$ de las infecciones progresan a enfermedad (7). Entre las descritas hasta el momento figuran la leucemia o linfoma de células $\mathrm{T}$, la paraparesia espástica tropical o miopatía asociada al HTLV, la uveítis, múltiples alteraciones dermatológicas, la miositis, la polimiositis, la polineuropatía, los trastornos psiquiátricos, las infecciones oportunistas, la infección por Strongyloides stercoralis y las condiciones reumatológicas autoinmunitarias (8-10). Se ha reportado la asociación entre el desarrollo de un tipo específico de enfermedad y la vía de transmisión del virus. Fujino, et al.,

\footnotetext{
Correspondencia:

Sandra Vargas, Banco de Sangre Fundación y Servicio Transfusional (sic), Clínica Valle del Lili, Avenida Simón Bolívar, Carrera 98 NN$^{\circ}$ 98-49, Santiago de Cali, Colombia

Teléfono: (572) 331 9090; extensiones 3248 y 3016

svargas@fcvl.org

Recibido: 30/06/15; aceptado: 10/02/16
}

concluyeron que el riesgo de desarrollar leucemia o linfoma de células T por infección con el HTLV es mayor cuando el virus se adquiere a través de la leche materna (11); Osame, et al., demostraron que existía un mayor riesgo para el desarrollo de paraparesia espástica tropical o miopatía asociada al HTLV en pacientes que habían adquirido el virus por transfusión sanguínea (12). La infección simultánea con el virus de la inmunodeficiencia humana (HIV) se presenta con frecuencia en áreas urbanas de Europa y África, donde se ha encontrado con mayor frecuencia en pacientes que tienen linfoma de células $T$ con complicaciones neurológicas, por lo cual se los considera en riesgo de complicaciones de mayor gravedad (13).

EI HTLV es un virus de distribución mundial, y se estima que 20 millones de personas están infectadas con él. Los países reportados como endémicos son Japón, países del centro y occidente de África, de Latinoamérica y de las islas del Caribe. Algunos estudios realizados en Suramérica reportan que la prevalencia del virus puede oscilar entre 0,05 y $7 \%(14-16)$.

En Colombia se han hecho pocos estudios sobre la prevalencia, la carga de las enfermedades y la dinámica de la trasmisión del virus. En dos estudios poblaciones en Tumaco, Nariño, en 1988, y en Puerto Tejada, Cauca, en 1997, se reportaron prevalencias de 7 y $3 \%$, respectivamente $(17,18)$. En otros estudios llevados a cabo en bancos de sangre del país se ha reportado que la seroprevalencia del virus en donantes puede oscilar entre 0,07 y $3,52 \%$ (19-21). Los datos adicionales reportados por la Red Nacional de Bancos de Sangre para el 2013 dan cuenta de una seroprevalencia de 0,21\%, similar a la reportada para el HIV, la cual fue de $0,23 \%(22)$.

A partir de 2014 es obligatorio para los bancos de sangre colombianos hacer pruebas de anticuerpos para los subtipos HTLV I y II en los donantes (23); sin embargo, en el Banco de Sangre de la Fundación Valle del Lili estas pruebas se vienen haciendo desde 1996, y a partir de 2012 se introdujo el Western blot como prueba confirmatoria. 
En este estudio se evaluaron los resultados de la tamización para el HTLV I y II en muestras de donantes de sangre en el periodo comprendido entre enero de 2008 y diciembre de 2014, con el objetivo de determinar la seroprevalencia de la respuesta reactiva al HTLV, evaluar su comportamiento temporal con la prueba de tamización utilizada y determinar la doble reacción frente a otros marcadores de infección en donantes del banco de sangre de la Fundación.

\section{Materiales y métodos}

Se hizo un estudio descriptivo de corte transversal en el banco de sangre de la Fundación. Se analizaron todos los registros de donantes de cualquier componente sanguíneo con prueba reactiva para anticuerpos IgG anti HTLV I-II, durante el periodo de 2008 a 2014.

Todos los donantes del banco de sangre de la Fundación son voluntarios. La captación de donantes se hace mediante diferentes estrategias y en diversos lugares (universidades, empresas, colegios y en la sede principal de la Fundación Valle del Lili). Todos los posibles donantes contestan un cuestionario del banco de sangre y se excluyen aquellos considerados de alto riesgo o quienes se rehúsan a la donación. Todas las unidades de sangre obtenidas se someten a tamización para HTLV I-II, enfermedad de Chagas, HIV I-II, sífilis, y virus de las hepatitis C y $\mathrm{B}$ (core HBsAg).

Las unidades de sangre del estudio se tamizaron para la detección de HTLV I y II con el método de inmunoensayo de quimioluminiscencia de micropartículas (CMIA), el cual se analizó mediante la plataforma automatizada Architect System $\left(A b b o t t^{\circledR}\right)$. A las muestras reactivas se les repitió la prueba de tamización dos veces y a las que fueron doblemente reactivas se les hizo la prueba confirmatoria mediante Western blot (MP Diagnostic HTLV BLOT 2.4). Ambas pruebas se hicieron siguiendo las especificaciones de los fabricantes y estuvieron a cargo de profesionales en bacteriología debidamente entrenados y certificados por Abbott Diagnostic Division.

Se contactó a todos los donantes con resultados confirmados mediante Western blot para ofrecerles asesoría y canalizarlos hacia la aseguradora de salud a la cual pertenecían, y cada caso fue reportado al Instituto Nacional de Salud. Las unidades de sangre con dos pruebas de tamización reactivas fueron incineradas, aquellas con una sola muestra reactiva y que posteriormente no lo fueron continuaron en el proceso de preparación de las unidades para la donación.

El Banco de Sangre de la Fundación Valle del Lili cuenta con un programa de garantía de calidad con controles de calidad internos y externos, estos últimos realizados por el Instituto Nacional de Salud, el programa de Medical Laboratory Evaluation y el del College of American Pathologists.

Aquellos registros en los cuales se reportaron otras pruebas de tamización reactivas para más de una enfermedad infecciosa, se consideraron como reactivos concomitantes para dos marcadores de infección.

\section{Análisis estadístico}

Se hizo un análisis univariado. Para determinar el comportamiento de las variables numéricas, se usó la prueba de Shapiro Wilk; aquellas con $p>0,05$ se consideraron con distribución normal y se presentaron con promedios y desviación estándar, y aquellas con distribución diferente a la normal se presentaron con mediana y rangos intercuartílicos como medida de resumen.

Las variables categóricas se expresaron como proporciones. La seroprevalencia acumulada de HTLV se calculó como una proporción, tomando como numerador el número total de donantes con prueba de tamización reactiva para HTLV y como denominador, a todos los donantes con prueba de tamización para HTLV durante el periodo del estudio. Entre 2012 y 2014 la seroprevalencia se ajustó mediante la prueba confirmatoria de Western blot. Se elaboró una línea de tendencia con los datos de las prevalencias calculadas por cada año para evaluar el comportamiento de la enfermedad durante el tiempo del estudio.

\section{Consideraciones éticas}

Este estudio se acogió a las normas internacionales de investigación del Council for International Organizations of Medical Sciences (CIOMS), a la Declaración de Helsinki y al Código de Nuremberg, así como a la normativa colombiana consignada en el artículo 11 de la Resolución 8430 de 1993. La investigación fue aprobada por el Comité de Ética en Investigación Biomédica de la Fundación Valle del Lili, según la carta de aprobación No. 209-2015.

\section{Resultados}

Entre enero de 2008 y diciembre de 2014 se evaluaron 77.117 registros de donantes a quienes 
se les aplicaron pruebas de tamización para anticuerpos anti-HTLV I-II, de los cuales 184 resultaron reactivos con el método de CMIA, cifra equivalente a una seroprevalencia acumulada de $0,24 \%$. La mediana de edad fue de 37 años (rango intercuartílico: 24-48) y la reacción positiva fue más frecuente en mujeres, con $61 \%$ de los donantes reactivos (cuadro 1).

Entre junio de 2012 y diciembre de 2014 se tamizaron 28.345 donantes, de los cuales 55 fueron doblemente reactivos para la prueba de tamización de CMIA, equivalente a una seroprevalencia de $0,23 \%$. De estas muestras, 27 fueron positivas en la prueba confirmatoria de Western blot, para una seroprevalencia ajustada de 0,10 \% (figura 1).

Las seroprevalencias desagregada por año fueron de: 0,13\% (2008), 0,19\% (2009), 0,31\% (2010), 0,32\% (2011), 0,38\% (2012), 0,14\% (2013) y $0,13 \%$ (2014). Las seroprevalencias ajustadas según los resultados en la prueba confirmatoria de Western blot entre 2012 y 2014 fueron de: $0,18 \%$ (2012) $0,08 \%$ (2013) y 0,07\% (2014) (figura 2).

La reacción positiva concomitante de HTLV I-II y otros marcadores de infección se presentó en $11 \%$ de los donantes. Los marcadores de sífilis fueron

Cuadro 1. Características demográficas de la población de estudio

\begin{tabular}{lrc}
\hline Variable & $\mathbf{n}$ & $\%$ \\
\hline Sexo & & \\
$\quad$ Hombres & 72 & 39 \\
$\quad$ Mujeres & 112 & 61 \\
Edad (años) & 52 & 28 \\
$18-25$ & 42 & 23 \\
$26-35$ & 36 & 20 \\
$36-45$ & 35 & 19 \\
$46-55$ & 19 & 10 \\
$56-65$ & & \\
Escolaridad & 34 & 18 \\
Primaria & 75 & 41 \\
Secundaria & 31 & 17 \\
Técnica & 37 & 20 \\
Universitaria & 1 & 1 \\
Posgrado & 6 & 3 \\
No determinada & & \\
Seguridad social & 29 & 16 \\
Subsidiado & 130 & 71 \\
Contributivo & 25 & 14 \\
No determinada & & \\
Estado civil & 87 & 47 \\
Soltero & 50 & 27 \\
Casado & 30 & 16 \\
Unión libre & 6 & 3 \\
Divorciado & 4 & 2 \\
Viudo & 7 & 4 \\
No determinado & & \\
\hline & & \\
\hline
\end{tabular}

Donantes del Banco de Sangre de la Fundación Valle de Lili, enero de 2008 a diciembre de $2014(n=77.117)$

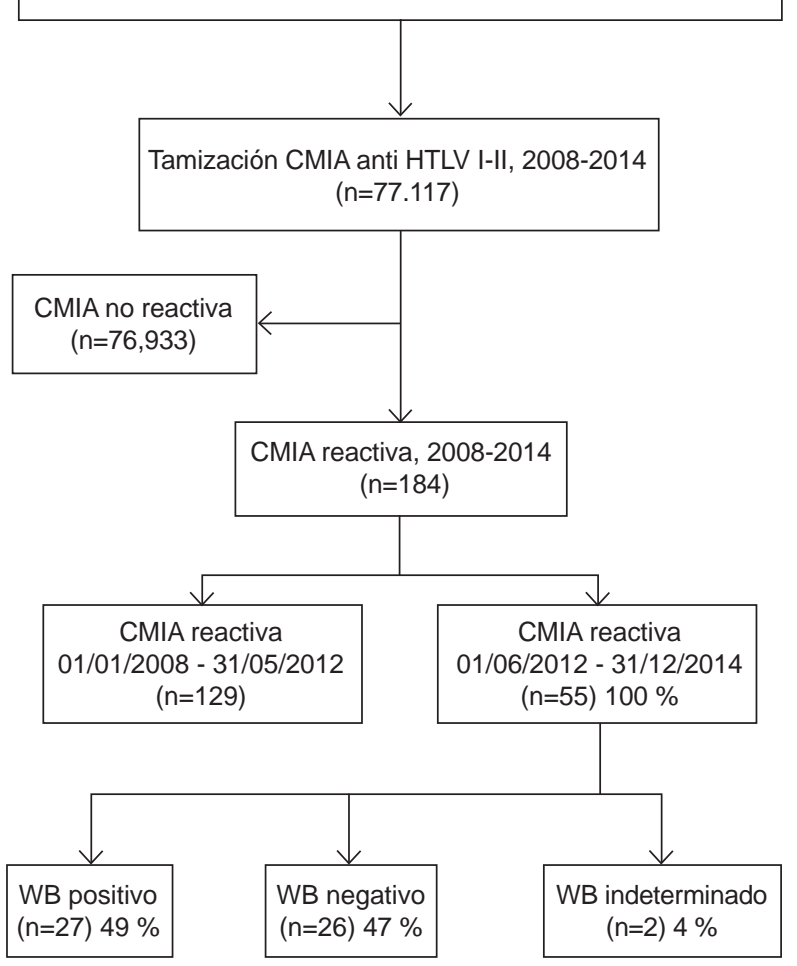

Figura 1. Flujograma del proceso de tamización y confirmación de HTLV I-II

CMIA: inmunoensayo quimioluminisciente de micropartículas WB: Western blot

los más frecuentes (57\%), seguidos del HIV (19\%), la hepatitis B $(14 \%)$ y la hepatitis C (9\%) (figura 3).

En cuanto a las características de la donación, $97 \%$ de las muestras reactivas en la prueba de tamización se habían obtenido por extracción de sangre total, $2 \%$ por extracción de plaquetas y $1 \%$ por transfusión de sangre autóloga. En relación con la clasificación $\mathrm{ABO}$ de los grupos sanguíneos, se encontró que $64 \%$ pertenecía al grupo O, $22 \%$ al $A, 11 \%$ al $B$, y $3 \%$ al $A B$, en tanto que $91,3 \%$ era Rh positivo.

\section{Discusión}

Entre el 5 y el $10 \%$ de las personas infectadas con HTLV I-II desarrollan alguna enfermedad relacionada con este virus (6). La infección por HTLV en individuos asintomáticos no tiene ningún tratamiento establecido y solo algunas de las enfermedades relacionadas con el virus lo tienen (24). El hecho de ser el causante de enfermedades graves, como la leucemia o el linfoma de células $T$ 


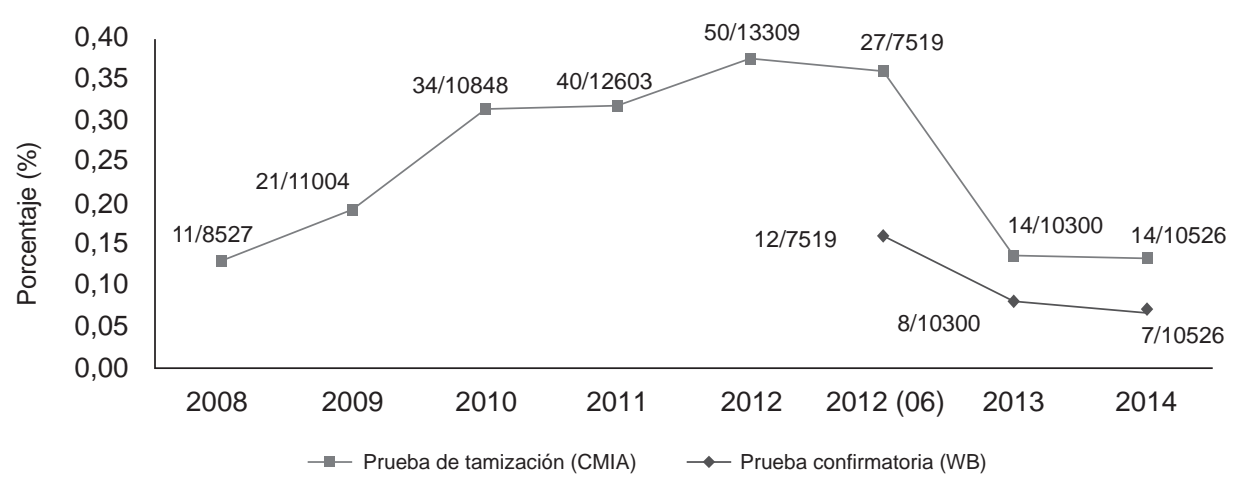

Figura 2. Comportamiento temporal de los resultados reactivos para HTLV en el inmunoensayo quimioluminisciente de micropartículas y positivos en el Western blot entre 2008 y 2014

El numerador representa el número total de pruebas reactivas por año. El denominador representa el número total de pruebas tomadas por año. BS-FVL: Banco de Sangre de la Fundación Valle del Lili; HTLV I-II: virus linfotrópico humano del tipo I-II; 2012 (06): periodo de tiempo entre el 1 de junio de 2012 y el 31 de diciembre de 2012; CMIA: inmunoensayo quimioluminisciente de micropartículas; WB: Western blot

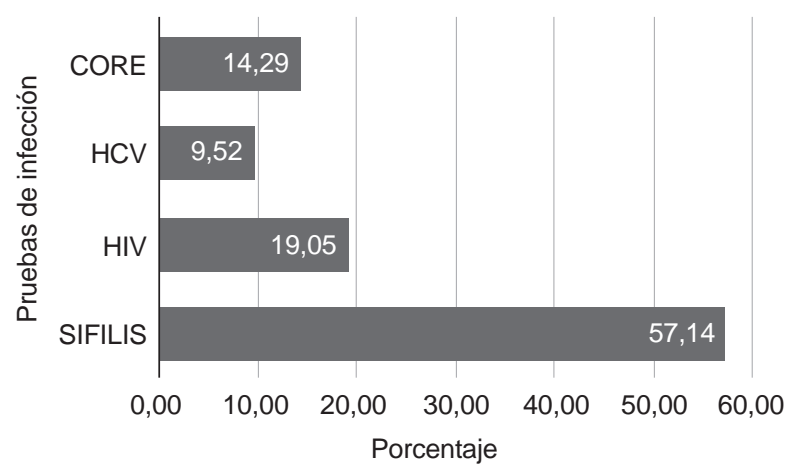

Figura 3. Resultados reactivos para marcadores de infección HIV: virus de inmunodeficiencia humana HCV: virus de la hepatitis C

y la paraparesia espástica tropical, ubica al HTLV en la categoría de los virus contra los cuales se trata de elaborar vacunas eficaces (25). Aunque algunos grupos de investigadores han trabajado arduamente en la elaboración de una vacuna, su labor ha sido infructuosa hasta el momento. En consecuencia, la única manera de prevenir las enfermedades relacionadas con el HTLV es evitar su contagio.

La transfusión de componentes de la sangre puede representar un importante riesgo de transmisión, especialmente en áreas endémicas en las cuales no se aplica la tamización para HTLV I-II (13). Los receptores de transfusiones infectados pueden ser portadores asintomáticos durante toda la vida, y son una fuente importante de transmisión del virus (24). En Colombia, la Red Nacional de Bancos de Sangre agrupa las unidades de servicios de transfusión en 26 departamentos, de los cuales solo 10 realizaban pruebas de tamización para HTLV I-II por estar ubicados en zonas endémicas para la transmisión. No obstante, ya en 1988, Cortés, et al., demostraron que la seroprevalencia del virus en zonas no endémicas era superior a la reportada en las zonas endémicas (21) y, a partir de febrero de 2014, el Ministerio de Salud y Protección Social estipuló como obligatorias para todos los bancos de sangre del país las pruebas de anticuerpos contra el HTLV I-II (Resolución 00437) (23).

El estudio reveló que la prevalencia acumulada de resultados reactivos en las pruebas de tamización para el HTLV I-II en el Banco de Sangre de la Fundación Valle del Lili fue de 0,24\%. En la mayoría de los países en donde se ha estudiado la seroprevalencia del virus, la distribución ha sido heterogénea y se ha encontrado una alta prevalencia en focos poblacionales pequeños y muy baja prevalencia en áreas cercanas (16). Es importante recalcar que el principal foco poblacional endémico registrado en el Banco de Sangre de la Fundación Valle del Lili es Puerto Tejada, Cauca, y, por lo tanto, su prevalencia resulta alta con respecto a lo esperado (18).

Al comparar los resultados de esta investigación con los estudios en bancos de sangre en los que solo se usaban pruebas de tamización, se encontró que en Bogotá, Bermúdez, et al., reportaron una seroprevalencia de 0,15\% (19), mucho menor a la encontrada en el Banco de Sangre de la Fundación Valle del Lili. En Caracas, Venezuela, se reportó una seroprevalencia similar a la encontrada en este estudio $(0,20 \%)(13)$. 
En el Banco de Sangre de la Fundación Valle del Lili, el $0,10 \%$ de las pruebas por Western blot fueron positivas. Al compararlo con otros bancos de sangre que realizan pruebas confirmatorias, se observó que, en Bogotá, Martínez, et al., encontraron $0,07 \%$ de resultados positivos, cifra menor a la reportada en este estudio (20). Comparada con la del estudio de Cortés, et al., la seroprevalencia en este fue mucho menor $(0,45 \%)$ (21), lo cual podría explicarse porque en el primero se incluyeron ocho bancos de sangre de diferentes ciudades colombianas (cuadro 2).

Algunos países han reportado seroprevalencias menores: Holanda, 0,002 \% (26), Estados Unidos, $0,001 \%$ (27) y Dinamarca, 0,003 \%. A diferencia de lo reportado por investigadores brasileros, quienes encontraron que la seroprevalencia se incrementaba con la edad (28-31), en esta investigación se registró una mayor prevalencia entre los menores de 30 años, en tanto que se estabilizó entre aquellos con mayor edad. En la literatura científica se ha reportado que una persona puede presentar anticuerpos detectables muchos años después de la infección (32), lo cual sugiere que la población objeto de investigación adquirió el HTLV en etapas tempranas de la vida (cuadro 3). Además, Castro, et al., encontraron que el mayor número de resultados reactivos frente a los marcadores de otras infecciones concomitantes con el HTLV se presentó con el virus de la hepatitis B (28), mientras que en el presente estudio la mayor concomitancia se presentó con el virus de la sífilis y el HIV. Las pruebas de tamización de los bancos de sangre son muy sensibles, por lo cual se presenta un gran número de falsos positivos. En este estudio, de los 55 donantes reactivos en la prueba de CMIA y de Western blot como prueba confirmatoria, 26 fueron falsos positivos.

En cuanto al comportamiento temporal de los resultados reactivos para HTLV I-II, la seroprevalencia aumentó de forma sucesiva desde el 2008 hasta el 2012, tendencia similar a la registrada en el estudio de Cruz, et al. (19). No se encontró asociación causal para este fenómeno, pues en

Cuadro 2. Estudios en bancos de sangre relacionados con la seroprevalencia de HTLV I-II

\begin{tabular}{|c|c|c|c|c|c|c|}
\hline Autores & Estudio & Técnica & Fecha & Banco de sangre & $\begin{array}{l}\text { Número de } \\
\text { muestras } \\
\text { analizadas }\end{array}$ & $\begin{array}{c}\text { Prevalencia } \\
(\%)\end{array}$ \\
\hline Zaninovic & $\begin{array}{l}\text { A propósito de } 5 \text { casos de } \\
\text { paraparesia espástica tropical } \\
\text { en Puerto Tejada (Cauca) }\end{array}$ & $\begin{array}{l}\text { Aglutinación de } \\
\text { partículas (PA, } \\
\text { Fujirebio Inc.) } \\
\text { y ELISA }\end{array}$ & 1997 & Población de Puerto Tejada, Cauca & 200 & 3 \\
\hline $\begin{array}{l}\text { Trujillo, } \\
\text { et al. }\end{array}$ & $\begin{array}{l}\text { Seroprevalence and cofactors } \\
\text { of HTLV-I infection in Tumaco, } \\
\text { Colombia. }\end{array}$ & & 1988 & & 1.077 & 2,8 \\
\hline \multirow{9}{*}{$\begin{array}{l}\text { Cortés, } \\
\text { et al. }\end{array}$} & \multirow{9}{*}{$\begin{array}{l}\text { Estudio prospectivo } \\
\text { seroepidemiológico de infección } \\
\text { por el virus linfotrópico humano } \\
\text { I y II (HTLV-I/II) en donantes de } \\
\text { sangre de áreas colombianas } \\
\text { endémicas y no endémicas }\end{array}$} & \multirow{9}{*}{$\begin{array}{l}\text { ELISA (Abbott) } \\
\text { y WB }\end{array}$} & \multirow[t]{9}{*}{1998} & Cruz Roja de Bogotá & 207 & 0,96 \\
\hline & & & & Hospital Regional de Leticia & 85 & 3,52 \\
\hline & & & & Hospital Universitario de Popayán & 165 & 0,6 \\
\hline & & & & Hospital San Pedro de Pasto & 160 & 1,25 \\
\hline & & & & Hospital Departamental de Buenaventura & 70 & 1,42 \\
\hline & & & & Hospital Civil de Ipiales & 90 & 1,11 \\
\hline & & & & Hospital Universitario de Cartagena & 81 & 1,23 \\
\hline & & & & Hospital San Andrés de Tumaco & 80 & 2,5 \\
\hline & & & & Global & 2.854 & 0,45 \\
\hline \multirow{5}{*}{$\begin{array}{l}\text { Bermúdez, } \\
\text { et al. }\end{array}$} & \multirow{5}{*}{$\begin{array}{l}\text { Seroprevalencia de tamización } \\
\text { frente a virus linfotrópico de } \\
\text { células T (HTLV) y factores } \\
\text { asociados a confección en } \\
\text { donantes voluntarios de sangre } \\
\text { de Colombia }\end{array}$} & \multirow{5}{*}{$\begin{array}{l}\text { MicroELISA } \\
\text { (ARCHITEC } \\
\text { I-2000 de } \\
\text { Abbott) }\end{array}$} & & \multirow{5}{*}{$\begin{array}{l}\text { Banco de Sangre Fundación } \\
\text { Hematológica Colombia }\end{array}$} & 93.377 & 0,08 \\
\hline & & & 2009 & & 98.396 & 0,12 \\
\hline & & & 2010 & & 100.658 & 0,28 \\
\hline & & & 2011 & & 141.494 & 0,23 \\
\hline & & & & & & 0,17 \\
\hline $\begin{array}{l}\text { Martínez, } \\
\text { et al. }\end{array}$ & $\begin{array}{l}\text { Seroprevalencia de anticuerpos } \\
\text { para virus linfotrópicos humanos } \\
\text { (HTLV I/II) en donantes de } \\
\text { sangre de una clínica de Bogotá, } \\
\text { Colombia, 1999-2004 }\end{array}$ & $\begin{array}{l}\text { ELISA de Murex } \\
\text { (ABBOTT) y } \\
\text { Western blot }\end{array}$ & $\begin{array}{l}1999- \\
2004\end{array}$ & Banco de Sangre Clínica Reina Sofía & 8.913 & 0,07 \\
\hline $\begin{array}{l}\text { León, } \\
\text { et al. }\end{array}$ & $\begin{array}{l}\text { Seropositividad al virus } \\
\text { linfotrópico de células T } \\
\text { humanas tipos I y II en donantes } \\
\text { del Banco Municipal de Sangre } \\
\text { de Caracas y factores de } \\
\text { riesgo asociados }\end{array}$ & ELISA (Abbott) & $\begin{array}{l}2000- \\
2001\end{array}$ & Banco Municipal de Sangre de Caracas & 23.413 & 0,20 \\
\hline
\end{tabular}


Cuadro 3. Distribución por grupos de edad y según sexo de los donantes reactivos en la prueba de CMIA

\begin{tabular}{|c|c|c|c|c|c|c|c|c|c|c|c|c|c|c|c|}
\hline \multirow{2}{*}{$\begin{array}{l}\text { Años } \\
\text { Sexo }\end{array}$} & \multicolumn{2}{|c|}{2008} & \multicolumn{2}{|c|}{2009} & \multicolumn{2}{|c|}{2010} & \multicolumn{2}{|c|}{2011} & \multicolumn{2}{|c|}{2012} & \multicolumn{2}{|c|}{2013} & \multicolumn{2}{|c|}{2014} & \multirow{2}{*}{$\begin{array}{c}\text { Total } \\
\text { general }\end{array}$} \\
\hline & $\mathbf{F}$ & M & $\mathbf{F}$ & M & $\mathbf{F}$ & M & $\mathbf{F}$ & M & $\mathbf{F}$ & M & $\mathbf{F}$ & M & $\mathbf{F}$ & M & \\
\hline $\begin{array}{l}\text { Rango de } \\
\text { edad (años) }\end{array}$ & \multicolumn{2}{|c|}{ n (\%) } & \multicolumn{2}{|c|}{ n (\%) } & \multicolumn{2}{|c|}{ n (\%) } & \multicolumn{2}{|c|}{ n (\%) } & \multicolumn{2}{|c|}{$n(\%)$} & \multicolumn{2}{|c|}{ n (\%) } & \multicolumn{2}{|c|}{ n (\%) } & \\
\hline $18-29$ & $1 \quad(9,2)$ & $2(18)$ & $2(10)$ & $4(19)$ & $9(26)$ & 3 (9) & $8(20)$ & 7 (18) & $16(32)$ & $8(16)$ & $3(21)$ & 1 (7) & $5(36)$ & 1 (7) & $70 \quad(38)$ \\
\hline $30-39$ & $2(18)$ & $1(9,2)$ & $1(5)$ & 1 (5) & $5(15)$ & $4(12)$ & $4(10)$ & $5(13)$ & 6 (12) & $3(6)$ & $1(7)$ & 1 (7) & $0(0)$ & $2(14)$ & $36 \quad(20)$ \\
\hline $40-49$ & $2(18)$ & $0 \quad(0)$ & $5(24)$ & $3(14)$ & $3(9)$ & $3(9)$ & $6(15)$ & $3(8)$ & $5(10)$ & $3(6)$ & 1 (7) & 1 (7) & $3(21)$ & $1(7)$ & 39 (21) \\
\hline $50-65$ & $2(18)$ & $1 \quad(9,2)$ & $3(14)$ & $2(10)$ & $5(15)$ & $2(6)$ & $4(10)$ & 3 (8) & 6 (12) & $3(6)$ & $2(14)$ & $4(29)$ & $2(14)$ & $0 \quad(0)$ & 39 (21) \\
\hline Total por sexo & $7(64)$ & $4(36)$ & $11(52)$ & $10(48)$ & $22(65)$ & $12(35)$ & $22(55)$ & $18(45)$ & $33(66)$ & $17(34)$ & $7(50)$ & $7(50)$ & $10(71)$ & $4(29)$ & \\
\hline Total general & & $1(100)$ & & $1(100)$ & & $4(100)$ & & $40(100)$ & & $0(100)$ & & $4(100)$ & & $4(100)$ & $184(100)$ \\
\hline
\end{tabular}

este intervalo de tiempo no hubo cambios en la entrevista que se hace a los donantes ni en las pruebas de tamización utilizadas.

El Banco de Sangre de la Fundación Valle del Lili capta $20 \%$ de todos los potenciales donantes del departamento del Valle, por lo tanto, los resultados de este estudio pueden extrapolarse a los donantes de los otros bancos de sangre de la ciudad, en la cual se ha estimado que uno de cada 500 donantes tendría una prueba de tamización reactiva para HTLV I-II. Dado que la población estudiada se seleccionó mediante una encuesta sobre los factores de riesgo para las enfermedades infecciosas, estos datos no representan la seroprevalencia de la población del suroccidente colombiano.

Los resultados de este estudio son un punto de partida para el desarrollo de futuros estudios poblacionales que amplíen el conocimiento sobre la dinámica de la trasmisión y la carga de las enfermedades asociadas a la infección por HTLV I-II. De igual manera, refuerza la necesidad de implementar y fortalecer las estrategias dirigidas a interrumpir la cadena de la trasmisión en las poblaciones en riesgo.

\section{Agradecimientos}

A Diana María Dávalos, por el apoyo constante en la labor investigativa de todos sus estudiantes y por su disposición a brindar soluciones ante las dificultades.

\section{Conflicto de intereses}

Todos los investigadores manifiestan no tener conflictos de intereses.

\section{Financiación}

Banco de Sangre y Centro de Investigaciones Clínicas de la Fundación Valle del Lili y Universidad ICESI.

\section{Referencias}

1. García-Vallejo F. Caracterización molecular y genómica del proceso de integración de provirus del virus linfotrópico humano (HTLV) tipo I. Rev Acad Colomb Cienc. 2006;30: 155-70.

2. Poiesz BJ, Ruscetti FW, Gazdar AF, Bunn PA, Minna JD, Gallo RC. Detection and isolation of type $C$ retrovirus particles from fresh and cultured lymphocytes of a patient with cutaneous T-cell lymphoma. Proc Natl Acad Sci. 1980;77:7415-9.

3. Kalyanaraman VS, Narayanan R, Feorino P, Ramsey RB, Palmer EL, Chorba T, et al. Isolation and characterization of a human T cell leukemia virus type II from a hemophilia-A patient with pancytopenia. EMBO J. 1985;4:1455-60.

4. Mahieux R, Gessain A. The human HTLV-3 and HTLV-4 retroviruses: New members of the HTLV family. Pathol Biol. 2009;57:161-6. http://dx.doi.org/10.1016/j.patbio.2008. 02.015

5. Gotuzzo-Herencia E, González-Lagos E, VerdonckBosteels K, Mayer-Arispe E, Ita-Nagy F, Clark-Leza D. Veinte años de investigación sobre HTLV-1 y sus complicaciones médicas en el Perú: perspectivas generales. Acta Médica Peru. 2010;27:196-203.

6. Proietti FA, Carneiro-Proietti AB, Catalan-Soares BC, Murphy EL. Global epidemiology of HTLV-I infection and associated diseases. Oncogene. 2005;24:6058-68. http:// dx.doi.org/10.1038/sj.onc.1208968

7. Gonçalves DU, Proietti FA, Ribas JG, Araújo MG, Pinheiro SR, Guedes AC, et al. Epidemiology, treatment, and prevention of human T-cell leukemia virus type 1-associated diseases. Clin Microbiol Rev. 2010;23:577-89. http://dx.doi.org/10.1128/CMR.00063-09.

8. Matsuura E, Umehara F, Nose H, Higuchi I, Matsuoka $\mathrm{E}$, Izumi $\mathrm{K}$, et al. Inclusion body myositis associated with human T-lymphotropic virus-type I infection: Eleven patients from an endemic area in Japan. J Neuropathol Exp Neurol. 2008;67:41-9. http://dx.doi.org/10.1097/nen. 0b013e31815f38b7

9. Tsukasaki K, Hermine O, Bazarbachi A, Ratner L, Ramos JC, Harrington W, et al. Definition, prognostic factors, treatment, and response criteria of adult T-cell leukemia-lymphoma: A proposal from an international consensus meeting. J Clin Oncol. 2009;27:453-9. http://dx. doi.org/10.1200/JCO.2008.18.2428 
10. Blank A, Rosso F. Hiperinfección por Strongyloides stercolaris en portadores del virus linfotrófico humano tipo I (HTLV-I). Acta Méd Colomb. 1996;21:122-6.

11. Fujino T, Nagata Y. HTLV-I transmission from mother to child. J Reprod Immunol. 2000;47:197-206.

12. Osame $\mathbf{M}$, Janssen $\mathbf{R}$, Kubota $\mathbf{H}$, Nishitani $\mathbf{H}$, Igata $\mathbf{A}$, Nagataki S, et al. Nationwide survey of HTLV-I-associated myelopathy in Japan: Association with blood transfusion. Ann Neurol. 1990;28:50-6.

13. León G, Quirós AM, López JL, Hung M, Díaz AM, Goncalves J, et al. Seropositividad al virus linfotrópico de células $T$ humanas tipos I y II en donantes del Banco Municipal de Sangre de Caracas y factores de riesgo asociados. Rev Panam Salud Pública. 2003;13:117-24. http://dx.doi.org/10.1590/S1020-49892003000200012

14. Chang YB, Kaidarova Z, Hindes D, Bravo M, Kiely $\mathbf{N}$, Kamel $\mathbf{H}$, et al. Seroprevalence and demographic determinants of human T-lymphotropic virus type 1 and 2 infections among first-time blood donors--United States, 2000-2009. J Infect Dis. 2013;209:523-31. http://dx.doi. org/10.1093/infdis/jit497

15. Alarcón-Villaverde J, Romaní-Romaní F, MontañoTorres S, Zunt JR. Vertical transmission of HTLV-1 in Perú. Rev Peru Med Exp Salud Pública. 2011;28:101-8. http://dx. doi.org/10.1590/S1726-46342011000100016

16. Gessain A, Cassar O. Epidemiological aspects and world distribution of HTLV-1 infection. Front Microbiol. 2012;3:388. http://dx.doi.org/10.3389/fmicb.2012.00388

17. Trujillo JM, Concha M, Muñoz A, Bergonzoli G, Mora C, Borrero I, et al. Seroprevalence and cofactors of HTLV-I infection in Tumaco, Colombia. AIDS Res Hum Retroviruses. 1992;8:651-7. http://dx.doi.org/10.1089/aid.1992.8.651

18. Zaninovic V, Moreno D, Payán C, Rodríguez A. A propósito de 5 casos de paraparesia espástica tropical en Puerto Tejada (Cauca). Colombia Médica. 1997;28:67-70.

19. Bermúdez HC, Enrique J, Collazos M, Sierra MR, Fonseca AA. Seroprevalencia de tamizaje frente a virus linfotrópico de células T (HTLV) y factores asociados a coinfección en donantes voluntarios de sangre de Colombia. Salud Uninorte. 2014;30:95-103.

20. Martínez-Nieto O, Isaza-Ruget M, Rangel-Espinosa N, Morales-Reyes OL. Seroprevalencia de anticuerpos para virus linfotrópicos humanos (HTLV I/II) en donantes de sangre de una clínica de Bogotá, Colombia. 1999-2004. Rev Salud Pública. 2007;9:253-61. http://dx.doi.org/10.1590/ S0124-00642007000200009

21. Buelvas AC, Beltrán M, Gallego GA, Isaza LM. Estudio prospectivo seroepidemiológico de infección por el virus linfotrópico humano I y II (HTLV-I/II) en donantes de sangre de áreas colombianas endémicas y no endémicas. Colombia Médica. 1999:30:19-25.
22. Red Nacional de Bancos de Sangre. Informe Nacional de Indicadores. 1 de enero a 31 de diciembre de 2013. Fecha de consulta: 21 de abril de 2015. Disponible en: http://www. ins.gov.co/lineas-de-accion/Red-Nacional-Laboratorios/ reas $\% 20$ Estratgicas/Informe\%20Anual\%20Red\%20 Sangre\%202013.pdf

23. Ministerio de Salud y Protección Social. Resolución número 000437. Fecha de consulta: 21 de abril de 2015. Disponible en: https://www.minsalud.gov.co/Normatividad Nuevo/Resoluci\%C3\%B3n\%200437\%20de\%202014.pdf

24. Gonçalves DU, Proietti FA, Ribas JG, Araújo MG, Pinheiro SR, Guedes AC, et al. Epidemiology, treatment, and prevention of human T-cell leukemia virus type 1-associated diseases. Clin Microbiol Rev. 2010;23:577-89. http://dx.doi.org/10.1128/CMR.00063-09

25. de Thé G, Bomford R. An HTLV-I vaccine: Why, how, for whom? AIDS Res Hum Retroviruses. 1993;9:381-6.

26. Zaaijer HL, Cuypers HT, Dudok de Wit C, Lelie PN. Results of 1-year screening of donors in The Netherlands for human T-lymphotropic virus (HTLV) type I: Significance of Western blot patterns for confirmation of HTLV infection. Transfusion. 1994;34:877-80. http://dx.doi.org/10.1046/j. 1537-2995.1994.341095026973.x

27. Centers for Disease Control and Prevention. Current trends human T-lymphotropic virus type I screening in volunteer blood donors, United States, 1989. MMWR Morb Mortal Wkly Rep. 1990;39:921-4.

28. Viana GM, Nascimento MdoD, de Oliveira RA, Dos Santos AC, Galvão CdeS, da Silva MA. Seroprevalence of HTLV-1/2 among blood donors in the state of Maranhão, Brazil. Rev Bras Hematol Hemoter. 2014;36:50-3. http://dx. doi.org/10.5581/1516-8484.20140013

29. Galvão-Castro B, Loures L, Rodriques LG, Sereno A, Ferreira-Júnior OC, Franco LG, et al. Distribution of human T-lymphotropic virus type I among blood donors: A nationwide Brazilian study. Transfusion. 1997;37:242-3. http://dx.doi.org/10.1046/j.1537-2995.1997.37297203532.x

30. Mota A, Nunes C, Melo A, Romeo M, Boasorte N, Dourado I, et al. A case-control study of HTLV-infection among blood donors in Salvador, Bahia, Brazil - associated risk factors and trend towards declining prevalence. Rev Bras Hematol Hemoter. 2006;28:120-6. http://dx.doi.org/10.1590/S151684842006000200011

31. Monteiro-de-Castro MS, Assunção RM, Proietti FA. Spatial distribution of the human T-lymphotropic virus types I and II (HTLV-I/II) infection among blood donors of Hemominas Foundation, Belo Horizonte, Minas Gerais State, Brazil, 1994-1996. Cad Saúde Pública. 2001;17:121930. http://dx.doi.org/10.1590/S0102-311X2001000500014

32. Navas MC, Iglesias A, Martínez BM, Caraballo L. Prevalencia de anticuerpos contra HTLV-1 en una población negra de Colombia. Biomédica. 1995;15:34-6. http://dx.doi. org/10.7705/biomedica.v15i1.855 